\title{
A Prospective Study Examining the Relationship Between Dispositional Mindfulness and Insomnia Among Male Prisoners in Iran: The Mediating Effect of Psychological Distress and Perceived Stress
}

\author{
Ali Poorebrahim ${ }^{1} \cdot$ Chung-Ying Lin $^{2}$ (D) Vida Imani ${ }^{3} \cdot$ Mark D. Griffiths $^{4}$ (D) \\ Amir H. Pakpour ${ }^{5,6}$ (D)
}

\begin{abstract}
Given the high stress and unfriendly environment caused by imprisonment, prisoners are at risk of developing insomnia. It is important to understand potential intervention (in this case, mindfulness) in improving insomnia for this population. The present study investigated whether dispositional mindfulness can be beneficial in helping relieve insomnia among Iranian prisoners. It also investigated whether psychological distress and perceived stress are potential mediators. Male prisoners $(\mathrm{N}=208)$ aged 39.08 years $(\mathrm{SD}=7.85)$ completed questionnaires and reported their demographic information and mindfulness (at baseline); psychological distress and sleep quality (at 1 month after baseline assessment); sleep quality and insomnia (at 3 months after baseline assessment). The mediation models using 10,000 bootstrapping resamples showed that sleep quality and insomnia significantly improved when mindfulness increased. Moreover, psychological distress and stress partially mediated the aforementioned association between dispositional mindfulness and sleep quality/insomnia. Using mindfulness to improve sleep quality/insomnia may be a promising psychological intervention for prisoners.
\end{abstract}

Keywords: insomnia; mindfulness; prisoners; psychological distress; sleep; stress 


\section{Introduction}

Prisoners are usually at high risk of developing health problems (Smith \& Braithwaite, 2016) due to the inherently stressful event of incarceration (Li \& Lai, 2019), and have been found to have high risk in developing insomnia compared to other populations (Dewa, Hassan, Shaw, \& Senior, 2015). Indeed, a recent UK study reported that the prevalence of insomnia was over 60\% (Dewa, Kyle, Hassan, Shaw, \& Senior, 2017). Among female prisoners, the prevalence can be even higher (up to 70.6\%; Dewa et al., 2017). Another recent study conducted in Taiwan also reported a relatively high prevalence of insomnia at $26.9 \%$ (Li \& Lai, 2019). Although the prevalence of insomnia among Taiwanese prisoners is lower than UK prisoners, the prevalence is higher than among Taiwanese general population $(\sim 5 \%)$ (Hsu et al., 2013). Other studies show similar findings that the prevalence of insomnia is higher among prisoners than among other populations (Dewa et al., 2015; Elger, 2004).

Several factors have been proposed as contributing to the high prevalence of insomnia among prisoners, including external environmental conditions (e.g., overcrowding), psychological reactions to incarceration (e.g., depressed mood), and problematic behaviors (e.g., unhealthy sleep hygiene behaviors) (Dewa et al., 2015; Elger, 2008). Indeed, the noise, light, overcrowding, lack of exercise, and boredom in prisons have been suggested as jeopardizing healthy sleep of individuals (Dewa et al., 2017; Elger, 2008). Therefore, developing effective interventions to treat insomnia among prisoners may be of interest to healthcare providers within the penal system.

One potential intervention treatment is mindfulness. There is evidence that mindfulness has some effectiveness in improving insomnia. For instance, mindfulness-based therapy for insomnia (MBTI), a meditation-based program that integrates behavioral techniques for insomnia, has been developed to treat the condition (Ong \& Sholtes, 2010; Ong, Shapiro, \& Manber, 2008; 2009). MBTI was adapted from mindfulness-based therapy for stress 
reduction and has been found to have promising effects in improving insomnia among adults with chronic insomnia (Ong et al., 2014). The use of MBTI overcomes the shortcomings expressed by patients receiving traditional treatments on insomnia. More specifically, patients receiving hypnotic medications have reported drug dependency, drug tolerance, and potential adverse effects, including residual daytime sleepiness, acute memory impairments, impaired balance, and poor gait (Morin, Gaulier, Barry, \& Kowatch, 1992; Vincent \& Lionberg, 2001).

The MBTI is an 8-week program, which begins with formal mindfulness meditations utilizing one quiet meditation (e.g., body scan, breathing, and sitting meditation) and one movement meditation (e.g., stretching, walking, and yoga meditation). Following this, the MBTI instructors (who were trained to lead a group discussion among participants) providing participants with the following directions: (i) the application of mindfulness principles to overcome insomnia problem, and (ii) the challenges in maintaining meditation practice (Ong et al., 2008; 2009; 2014). The MBTI also incorporates specific behavioral strategies within the context of mindfulness principles for insomnia; for example, sleep restriction therapy (Spielman, Saskin, \& Thorpy, 1987), sleep hygiene behaviors (Strong et al., 2018), and stimulus control (Bootzin \& Epstein, 2011). The 8-week MBTI additionally instructs participants to practice meditation at home for 30-45 minutes, six days per week. The participants are requested to keep a meditation diary and a sleep diary to monitor their adherence and progress (Ong et al., 2008; 2009; 2014). Moreover, a recent meta-analysis showed that mindfulness-based therapy was found to be effective in improving symptoms of insomnia and sleep quality among 13 trials that have been published since 2010 (Rash, Kavanagh, \& Garland, 2019). The 13 trials were implemented across diverse populations, such as veterans, cancer survivors, individuals with fibromyalgia, and postmenopausal women, but none of these trials utilized prisoners as participants.

Finally, a systematic review of the efficacy of Buddhist-derived interventions (BDIs; 
i.e., mindfulness and meditation) among eight studies of incarcerated individuals by Shonin, Van Gordon, Slade and Griffiths (2013) demonstrated there were significant improvements among prisoners in five key domains (i.e., negative affect, substance use, anger/hostility, relaxation capacity, and self-esteem/optimism). They concluded that BDIs may be effective as rehabilitative interventions for prisoners.

Although MBTI is effective among different populations with sleep problems, it is uncertain whether MBTI or mindfulness-related treatment has similar effects on prisoners given the lack of empirical studies. Therefore, studies are needed to understand this issue and the present study examined the relationship between dispositional mindfulness and insomnia among prisoners. Moreover, previous research has shown that mindfulness has positive psychological effects; that is, mindfulness is negatively related to stress and psychological distress (Keng, Smoski, \& Robins, 2011). Psychological distress and stress are potential factors in developing insomnia (Lin et al., 2019; Rajalingam, Jacobsen, Nielsen, Einarsen, \& Gjerstad, 2019; Shaver, Johnston, Lentz, \& Landis, 2002), and these are proposed as being potential mediators in the relationship between mindfulness and insomnia. An investigation into such meditational mechanisms will help healthcare providers determine whether mindfulness-based treatment or MBTI would be of utility for prisoners to help overcome their sleep problems. More specifically, healthcare providers can understand why MBTI is effective and use the information to motivate prisoners to receive MBTI.

Although no empirical evidence has been reported regarding MBTI on prisoners' insomnia, prior literature has demonstrated positive effects of mindfulness-based therapy on other health outcomes among prisoners. For example, Ifeagwazi, Nwokpoku, Chukwuorji et al. (2019) found that the higher level of mindfulness is, the lower somatic symptoms are among 209 prison inmates. The effects of mindfulness-based therapy have also been demonstrated on the reduction of psychological distress among 59 female prisoners (Riley, 
Smith, \& Baigent, 2019). Similarly, An et al. (2019a) reported the beneficial effects of mindfulness-based therapy on mental health of 19 male prisoners. An and colleagues (2019b) further found that mindfulness-based therapy had larger effects on mental health among male prisoners serving longer-term sentences in the prison than those with shorter-term ones. Moreover, a recent systematic review and meta-analysis shows that mindfulness-based therapy incorporated with yoga improved psychological wellbeing and behavioral functioning among prisoners (Auty, Cope, \& Liebling, 2017). However, to the best of the present authors' knowledge, no evidence on the association between mindfulness and sleep has previously been reported among prisoners. Consequently, the present study examining the relationship between mindfulness and insomnia among prisoners addressed this gap in the literature.

Using a prospective design across a three-month period, the present study investigated (i) whether dispositional mindfulness can be beneficial in improving insomnia/sleep quality among Iranian prisoners, and (ii) whether the association found between dispositional mindfulness and insomnia/sleep quality can be mediated by psychological distress and perceived stress among Iranian prisoners.

\section{Methods}

\section{Participants and procedure}

This prospective study comprised a sample of male prisoners in Lakan Prison (Guilan province, Iran) that were recruited between February 2019 and January 2020. Lakan prison is located $13 \mathrm{~km}$ from Rasht city center. The total number of prisoners was approximately 3200 . Prisoners were eligible if they were aged 18 years or over, were able to communicate in Persian, and had been incarcerated within the facility for at least 6 months. Prisoners were excluded if they were unable to give consent, were sentenced to death, were profoundly intellectually disabled, or too mentally ill. Prisoners' eligibility was determined by the first 
author. The total sample size was 208 prisoners. The study was approved by the ethics committee of Qazvin University of Medical Sciences (IR.QUMS.REC.1397.294) and Prisons and Security and Corrective Measures Organization (70/31/13). Mindfulness and sociodemographic variables were assessed at baseline. Psychological distress and stress were assessed at 1 month after baseline assessment. Sleep quality and insomnia were assessed 3 months after the baseline assessment.

\section{Measures}

Demographics. Demographic data (including age, year of education, marital status, having children or not, occupational status, and histories of drug and alcohol abuse) were gathered using a background information sheet.

Sleep quality. Sleep quality/disturbance was assessed using the Pittsburgh Sleep Quality Index (PSQI). The PSQI comprises 19 self-rated items that can be converted to seven components, and each component is scored on a four-point Likert scale (from 0 to 3 ). The seven components can be summed to provide a global PSQI score, ranging between 0 and 21, where a higher score indicates poor sleep quality. The psychometric properties of the Persian PSQI have been supported by good internal consistency $(\alpha=0.77)$ (Moghaddam, Nakhaee, Sheibani, Garrusi, \& Amirkafi, 2012). Cronbach's alpha in the present study was 0.86 . Insomnia. The severity and effects of insomnia were assessed using the 7-item Insomnia Severity Index (ISI). All the ISI items are rated on a five-point Likert scale with a total score ranging between 0 and 28 after summing up all responses. Different levels of insomnia have been defined using the total score: 0-7 indicates absence of insomnia, 8-14 indicates sub-threshold insomnia, 15-21 indicates moderate insomnia, and 22-28 indicates severe insomnia (Morin, Belleville, Bélanger, \& Ivers, 2011). Consequently, a higher ISI score indicates more severe insomnia. The psychometric properties of the Persian ISI were very good in terms of its internal consistency ( $\alpha=0.82$ and 0.87 ), test-retest reliability (intraclass 
correlation coefficient $=0.84)$, and convergent validity ( $r=0.64$ with Athens Insomnia Scale) (Lin et al., 2020; Yazdi, Sadeghniiat-Haghighi, Zohal, \& Elmizadeh, 2012). Cronbach's alpha in the present study was 0.91 .

Psychological distress. Psychological distress (i.e., anxiety and depression) was assessed using Hospital Anxiety and Depression Scale (HADS). The HADS comprises 14 self-rated items that are rated on a four-point Likert scale (from 0 to 3). The 14 item responses can be summed up after some items are reverse-scored. A global psychological distress score, ranging between 0 and 42 , can be generated with a higher score indicating greater distress. The psychometric properties of the Persian HADS have been supported by good internal consistency ( $\alpha=0.82$ in Anxiety subscale and 0.79 in Depression subscale) (Lin \& Pakpour, 2017). Cronbach's alpha in the present study was 0.80 .

Stress. Stress was assessed using the 10-item Perceived Stress Scale (PSS). All the PSS items are rated on a five-point Likert-type scale (from 0 to 4 ) with a total PSS score ranging between 0 and 40. A higher PSS score indicates a higher level of stress. The psychometric properties of the Persian PSS have been supported by excellent internal consistency $(\alpha=0.90)$ and good convergent validity $(\mathrm{r}=0.71$ with Stress subscale in the Depression, Anxiety, Stress Scale-21) (Maroufizadeh, Zareiyan, \& Sigari, 2014). Cronbach's alpha in the present study was 0.83 .

Mindfulness. Dispositional mindfulness was assessed using the Mindful Attention Awareness Scale (MAAS), comprising 15 items assessing individual differences in the level of dispositional mindfulness and is one of the commonly used instruments to assessing mindfulness (Brown \& Ryan, 2003; Medvedev et al., 2015). All the MAAS items are rated on a 6-point Likert-type scale (from 1 to 6) with a total MAAS score ranging between 15 and 90. A higher MAAS score indicates a higher level of dispositional mindfulness. The psychometric properties of the Persian MAAS have been supported by good internal 
consistency $(\alpha=0.76)$ and construct validity (Abdi, Ghabeli, Abbasiasl, \& Shakernagad, 2015). Cronbach's alpha in the present study was 0.88 .

\section{Data analysis}

Apart from the descriptive statistics on participants' characteristics, Pearson correlations were conducted between the study variables, including sleep quality (assessed using PSQI), insomnia (assessed using ISI), psychological distress (assessed using HADS), stress (assessed using PSS), and mindfulness (assessed using MAAS). In terms of the mediation models, sleep quality and insomnia were treated as dependent variables; psychological distress and stress as mediators; and dispositional mindfulness as the independent variable (Figure 1). The mediation models were analyzed using the Model 4 in the PROCESS macro for SPSS with 10,000 bootstrapping resamples (Model 4, Process Macro; Hayes, 2013).

\section{Results}

The characteristics of the sample are shown in Table 1. More specifically, the mean age of the 208 male prisoners was 39.08 years $(\mathrm{SD}=7.85)$ years and the mean number of years of education was 9.58 years $(\mathrm{SD}=3.01)$. More than half of the participants were married $(58.2 \%)$; had children (53.4\%); were self-employed (55.3\%); and had a history of alcohol abuse (58.5\%). The majority of the participants had a history of drug abuse $(77.9 \%)$.

(Insert Table 1 here)

Table 2 demonstrates the correlations between, psychological distress, sleep quality, insomnia, stress, and dispositional mindfulness among the participants (absolute $r=0.380$ to $0.675 ; p<0.01)$. More specifically, mindfulness was negatively correlated with all the other variables. All the variables, except for dispositional mindfulness, were mutually positively correlated. After ensuring that all the correlations among the variables were significant, mediation analyses were carried out using regression models with bootstrapping methods. 
The total effect of dispositional mindfulness was $-0.144(\mathrm{SE}=0.021 ; \mathrm{t}=6.73 ; p<0.001)$ on sleep quality and was $-0.303(\mathrm{SE}=0.032 ; \mathrm{t}=9.47 ; p<0.001)$ on insomnia. In short, sleep quality significantly improved and insomnia significantly reduced when dispositional mindfulness increased.

Additionally, psychological distress and stress both significantly mediated the effects of dispositional mindfulness on sleep quality and on insomnia - as shown by the lower and upper limits of the confidence interval in the bootstrapping samples (see Table 3). In addition, mindfulness had significant direct effects on both sleep quality and insomnia (Table 3). Therefore, psychological distress and stress partially mediated the effects of dispositional mindfulness on sleep quality and insomnia.

(Insert Tables 2 and 3 here)

\section{Discussion}

The present study is, to the best of the authors' knowledge, the first study that adopts a prospective design to investigate the meditating relationships between dispositional mindfulness and insomnia/sleep quality with potential mediators (psychological distress and stress) among a sample of prisoners. More specifically, the present study demonstrated that there were direct temporal associations between dispositional mindfulness and insomnia/sleep quality. Moreover, both psychological distress and stress were significant mediators in the association between dispositional mindfulness and insomnia/sleep quality. This corresponds with previous research showing that mindfulness is negatively related to stress and psychological distress (Keng et al., 2011) and that psychological distress and stress are potential factors in developing insomnia (Lin et al., 2019; Rajalingam et al., 2019; Shaver et al., 2002). The findings of the present study further suggest the existence of both mediators in the association between dispositional mindfulness and insomnia/sleep quality. Moreover, 
the findings extend the evidence base from general population (or population with insomnia) to a specific population (i.e., prisoners).

The findings of present study also concur with prior findings concerning the associations between mindfulness and mental health (An et al., 2019a; 2019b; Auty et al., 2017; Ifeagwazi et al., 2019; Riley et al., 2019). More specifically, mindfulness-based intervention was found to be effective in improving psychological distress, psychological wellbeing, and behavioral functioning among prisoners (An et al., 2019a; 2019b; Auty et al., 2017; Riley et al., 2019), which indicates that higher mindfulness was associated with lower psychological distress and better psychological wellbeing and behavioral functioning. Therefore, the relationships between mindfulness, psychological distress, and perceived stress found in the present study are in line with prior findings. The present study's findings also concur with findings concerning the relationships between psychological distress, stress, and insomnia (Lin et al., 2019; Rajalingam et al., 2019; Shaver et al., 2002). More specifically, psychological distress and stress are proposed to be potential contributors to insomnia development (Lin et al., 2019; Rajalingam et al., 2019; Shaver et al., 2002). Therefore, the associations between psychological distress, perceived stress, and insomnia found in the present study somewhat support the notion that psychological distress and stress may result in insomnia.

Previous literature has documented the evidence regarding the negative effects of insomnia on health, especially psychological health, such as unstable mood and high stress (Anothaisintawee, Reutrakul, Van Cauter, \& Thakkinstian, 2016; Sivertsen, Krokstad, Øverland, \& Mykletun, 2009; Sofi et al., 2014). Therefore, treating insomnia for prisoners is important given that it can further help society to decrease the burden in taking care of the negative consequences (i.e., poor health) resulting from insomnia/sleep problems. Moreover, the potential treatment effects of MBTI for prisoners are indicated by the negative association found between dispositional mindfulness and insomnia positive association. The positive 
association between dispositional mindfulness and sleep quality also supports the MBTI effects. These findings also correspond to a series of studies that reported the promising effects of MBTI in improving insomnia/sleep quality (Ong et al., 2008; 2009; 2010; 2014). However, given that the present study is not an intervention study; further evidence is needed to ensure the effectiveness of MBTI for treating insomnia problems among prisoners. Nevertheless, the findings in the present study can be viewed as a starting point to design MBTI for prisoners.

Several implications are proposed based on the present study's findings. First, the mediated effects found in the present study together with prior findings regarding the effectiveness of mindfulness-based therapy on prisoners' mental health (An et al., 2019a; 2019b; Auty et al., 2017; Ifeagwazi et al., 2019; Riley et al., 2019) suggest healthcare providers should consider introducing mindfulness-based therapy to ease psychological distress among prisoners. With the improvement of psychological distress, sleep problems among prisoners may be minimized. Second, given that the present study demonstrated the direct effect between mindfulness and insomnia/sleep quality, healthcare providers may consider using MBTI directly to improve insomnia and/or sleep quality for prisoners. Third, insomnia and sleep quality may be directly improved by mindfulness and indirectly improve psychological distress and stress. Therefore, healthcare providers should consider providing MBTI and mindfulness-based therapy for psychological distress (or on stress) simultaneously. Consequently, prisoners' sleep may be improved via two routes: a direct route from mindfulness on sleep and an indirect route from the improved psychological distress (or stress).

\section{Limitations and strengths}

There are a number of limitations in the present study. First, all the instruments used in the study were self-report. Therefore, the present study suffers from the common biases 
resulting from subjective measures, including social desirability (i.e., the participants try to hide their weakness and intend to report their positive performance) and recall biases (e.g., the participants may have had difficulties in reporting their sleep time when completing the PSQI). However, it was assumed that all the instruments used in the present study were valid and trustworthy given the strong and robust psychometric properties shown in these instruments (Abdi et al., 2015; Brown \& Ryan, 2003; Lin et al., 2020; Lin \& Pakpour, 2017; Maroufizadeh et al., 2014; Medvedev et al., 2015; Moghaddam et al., 2012; Morin et al., 2011; Yazdi et al., 2012). Nevertheless, future studies are recommended that adopt additional objective measures where appropriate (e.g., an Actigraph that can assess sleep objectively) to provide additional evidence to the proposed mediation model presented here. Second, the generalizability of the present study's findings is restricted to Iranian prisoners because the sample comprised incarcerated males from the same prison). Therefore, the present study's findings may be hard to generalize to all of Iran, or the populations in other countries. Third, although the prospective design supports a potential causal effect among the factors of mindfulness, insomnia, sleep quality, psychological distress, and stress, stronger evidence is needed using randomized controlled trials. More specifically, randomized controlled trials can help us understand whether the improved mindfulness leads to stress/psychological distress reduction and insomnia/sleep quality improvement.

The strength of the present study includes the relatively large sample size for this type of target population (over 200 adult prisoners) and the robust statistical testing. The sample size decreases the potential biases that could be contributed by any outliers, and the robust statistical testing indicates the high internal validity for the present study. Therefore, although the present study had the weakness in the representativeness of study participants, there is confidence that the findings (i.e., dispositional mindfulness, psychological distress, and perceived stress are predictors of insomnia/sleep quality) can be generalized to Iranian 
prisoners who share similar characteristics to the sample investigated here. Additionally, this is, to the best of the authors' knowledge, the first study to adopt a prospective design to better understand whether psychological distress and perceived stress are mediators in the association between mindfulness and insomnia/sleep quality.

\section{Conclusion}

In conclusion, positive effects of dispositional mindfulness on improving insomnia/sleep quality and on reducing psychological distress/stress among Iranian prisoners were found in the present study. Additionally, psychological distress and perceived stress were significant mediators in the effects of dispositional mindfulness on insomnia/sleep quality improvement. Therefore, using mindfulness to reduce psychological distress and stress may be therapeutically important for prisoners in tackling their insomnia/sleep problems. Therefore, future studies are recommended to examine the effects of improving mindfulness, reducing psychological distress, and decreasing perceived stress among prisoners. 


\section{References}

Auty, K. M., Cope, A., \& Liebling, A. (2017). A Systematic review and meta-analysis of yoga and mindfulness meditation in prison. International Journal of Offender Therapy and Comparative Criminology, 61, 689-710. doi: 10.1177/0306624X15602514.

Abdi, S., Ghabeli, F., Abbasiasl, Z., \& Shakernagad, S. (2015). Mindful Attention Awareness Scale (MAAS): Reliability and validity of Persian version. Journal of Applied Environmental and Biological Sciences, 4, 43-47.

Anothaisintawee, T., Reutrakul, S., Van Cauter, E., \& Thakkinstian, A. (2016). Sleep disturbances compared to traditional risk factors for diabetes development: systematic review and meta-analysis. Sleep Medicine Review, 30, 11-24.

https://doi.org/10.1016/j.smrv.2015.10.002.

An, Y., Huang, Q., Zhou, Y., Zhou, Y., \& Xu, W. (2019b). Who can get more benefits? Effects of mindfulness training in long-term and short-term male prisoners. International Journal of Offender Therapy and Comparative Criminology, 63, 2318-2337. doi: $10.1177 / 0306624 X 19846771$.

An, Y., Zhou, Y., Huang, Q., Jia, K., Li, W., \& Xu, W. (2019a). The effect of mindfulness training on mental health in long-term Chinese male prisoners. Psychology, Health, and Medicine, 24, 167-176. doi: 10.1080/13548506.2018.1510130.

Bootzin, R. R., \& Epstein, D. R. (2011). Understanding and treating insomnia. Annual Review of Clinical Psychology, 7, 435-458. doi:

10.1146/annurev.clinpsy.3.022806.091516

Brown, K. W., \& Ryan, R. M. (2003). The benefits of being present: mindfulness and its role in psychological well-being. Journal of Personality and Social Psychology, 84, 822-848. doi: $10.1037 / 0022-3514.84 .4 .822$

Dewa, L. H., Hassan, L., Shaw, J. J., \& Senior, J. (2017). Trouble sleeping inside: a 
cross-sectional study of the prevalence and associated risk factors of insomnia in adult prison populations in England. Sleep Medicine, 32, 129-136.

doi:10.1016/j.sleep.2016.12.018

Dewa, L. H., Kyle, S. D., Hassan, L., Shaw, J., \& Senior, J. (2015). Prevalence, associated factors and management of insomnia in prison populations: an integrative review. Sleep Medicine Review, 24, 13-27. doi: 10.1016/j.smrv.2014.12.002.

Elger, B. S. (2004). Prevalence, types and possible causes of insomnia in a Swiss remand prison. European Journal of Epidemiology, 19, 665-677. doi:

10.1023/b:ejep.0000036805.58237.e3

Elger, B. S. (2008). Prisoners' insomnia: to treat or not to treat? Medical decision-making in places of detention. Medicine, Science and the Law, 48, 307-316. doi: $10.1258 /$ rsmmsl.48.4.307

Hsu, Y. W., Ho, C. H., Wang, J. J., Hsieh, K. Y., Weng, S. F., \& Wu, M. P. (2013). Longitudinal trends of the healthcare-seeking prevalence and incidence of insomnia in Taiwan: An 8-year nationally representative study. Sleep Medicine, 14(9), 843-849. doi: 10.1016/j.sleep.2013.02.017.

Ifeagwazi, C. M., Nwokpoku, E. E., Chukwuorji, J. C., Eze, J. E., \& Abiama, E. E. (2019). Somatic symptoms among prison inmates: contributions of emotion regulation, dispositional mindfulness, and duration of stay in prison. International Journal of Prisoner Health. Advance online publication. doi: 10.1108/IJPH-02-2019-0009.

Keng, S. L., Smoski, M. J., \& Robins, C. J. (2011). Effects of mindfulness on psychological health: A review of empirical studies. Clinical Psychology Review, 31, 1041-1056. doi: 10.1016/j.cpr.2011.04.006.

Li, M. R., \& Lai, H. L. (2019). Insomnia and its associated factors in incarcerated adults: a cross-sectional study. Nursing \& Health Sciences, 21(3), 330-335. doi: 
10.1111/nhs. 12600 .

Lin, C.-Y., Imani, V., Broström, A., Huus, K., Björk, M., Hodges, E. A., Pakpour, A. H. (2019). Psychological distress and quality of life in Iranian adolescents with overweight/obesity: Mediating roles of weight bias internalization and insomnia. Eating \& Weight Disorders. 2019. doi: 10.1007/s40519-019-00795-5. [Epub ahead of print]

Lin, C.-Y., \& Pakpour, A, H. (2017). Using Hospital Anxiety and Depression Scale (HADS) on patients with epilepsy: Confirmatory factor analysis and Rasch models. Seizure, 45, 42-46. doi: 10.1016/j.seizure.2016.11.019.

Lin, C.-Y., Cheng, A. S. K., Nejati, B., Imani, V., Ulander, M., Browall, M., ..., Pakpour, A. H. (2020). A thorough psychometric comparison between Athens Insomnia Scale and Insomnia Severity Index among patients with advanced cancer. Journal of Sleep Research, 29(1), e12891. doi: 10.1111/jsr.12891.

Maroufizadeh, S., Zareiyan, A., \& Sigari, N. (2014). Reliability and validity of Persian version of perceived stress scale (PSS-10) in adults with asthma. Archives of Iranian Medicine, 17(5), 361-365. doi: 0141705/AIM.0010.

Medvedev, O.N., Siegert, R.J., Feng, X.J., Billington, D.R., Jang, J.Y., Krägeloh, C.U. (2016). Measuring trait mindfulness: How to improve the precision of the mindful attention awareness scale using a Rasch model. Mindfulness, 7, 384-395. https://doi.org/10.1007/s12671-015-0454-z

Moghaddam, J. F., Nakhaee, N., Sheibani, V., Garrusi, B., \& Amirkafi, A. (2012). Reliability and validity of the Persian version of the Pittsburgh Sleep Quality Index (PSQI-P). Sleep \& Breathing, 16, 79-82. doi: 10.1007/s11325-010-0478-5.

Morin, C. M., Belleville, G., Bélanger, L., \& Ivers, H. (2011). The Insomnia Severity Index: Psychometric indicators to detect insomnia cases and evaluate treatment response. Sleep 34(5), 601-608. doi: 10.1093/sleep/34.5.601 
Morin, C. M., Gaulier, B., Barry, T., \& Kowatch, R. A. (1992). Patients' acceptance of psychological and pharmacological therapies for insomnia. Sleep, 15, 302-305. doi: 10.1093/sleep/15.4.302

Ong, J., \& Sholtes, D. (2010). A mindfulness-based approach to the treatment of insomnia. Journal of Clinical Psychology, 66, 1175-1184. doi: 10.1002/jclp.20736.

Ong, J. C., Manber, R., Segal, Z., Xia, Y., Shapiro, S., \& Wyatt, J. K. (2014). A randomized controlled trial of mindfulness meditation for chronic insomnia. Sleep, 37(9), 1553-1563. doi: $10.5665 /$ sleep.4010.

Ong, J. C., Shapiro, S. L., \& Manber, R. (2008). Combining mindfulness meditation with cognitive-behavior therapy for insomnia: A treatment-development study. Behavior Therapy, 39, 171-182. doi: 10.1016/j.beth.2007.07.002.

Ong, J. C., Shapiro, S. L., \& Manber, R. (2009). Mindfulness meditation and cognitive behavioral therapy for insomnia: a naturalistic 12-month follow-up. Explore, 5, 30-36. doi: 10.1016/j.explore.2008.10.004.

Rajalingam, D., Jacobsen, D. P., Nielsen, M. B., Einarsen, S. V., \& Gjerstad, J. (2019). Exposure to workplace bullying, distress, and insomnia: The moderating role of the mir-146a genotype. Frontiers in Psychology, 10, 1204. doi:10.3389/fpsyg.2019.01204

Rash, J. A., Kavanagh, V. A. J., \& Garland, S. N. (2019). A meta-analysis of mindfulness-based therapies for insomnia and sleep disturbance: Moving towards processes of change. Sleep Medicine Reviews, 14, 209-233. doi: 10.1016/j.jsmc.2019.01.004.

Riley, B. J., Smith, D., \& Baigent, M. F. (2019). Mindfulness and acceptance-based group therapy: An uncontrolled pragmatic pre-post pilot study in a heterogeneous population of female prisoners. International Journal of Offender Therapy and Comparative Criminology, 63, 2572-2585. doi: 10.1177/0306624X19858487. 
Shaver, J. L., Johnston, S. K., Lentz, M. J., \& Landis, C. A. (2002). Stress exposure, psychological distress, and physiological stress activation in midlife women with insomnia. Psychosomatic Medicine, 64(5), 793-802. doi:

10.1097/01.psy.0000024235.11538.9a

Shonin, E. S., Van Gordon, W., Slade, K. \& Griffiths, M. D. (2013). Mindfulness and other Buddhist-derived interventions in correctional settings: A systematic review. Aggression and Violent Behavior, 18, 365-372. doi: 10.1016/j.avb.2013.01.002

Sivertsen, B., Krokstad, S., Øverland, S., \& Mykletun, A. (2009). The epidemiology of insomnia: associations with physical and mental health: the HUNT-2 study. Journal of Psychosomatic Research, 67, 109-116. https://doi.org/10.1016/j.jpsychores.2009.05.001.

Smith, S. A., \& Braithwaite, R. L. (2016). Introduction to public health and incarceration: social justice matters. Journal of Health Care for the Poor and Underserved, 27(2A), 1-4. doi: 10.1353/hpu.2016.0048.

Sofi, F., Cesari, F., Casini, A., Macchi, C., Abbate, R., \& Gensini, G. F. (2014). Insomnia and risk of cardiovascular disease: a meta-analysis. European Journal of Preventive Cardiology, 21(1), 57-64. https://doi.org/10.1177/2047487312460020.

Spielman, A. J., Saskin, P., \& Thorpy, M. J. (1987). Treatment of chronic insomnia by restriction of time in bed. Sleep, 10, 45-56. https://doi.org/10.1093/sleep/10.1.45

Strong, C., Lin, C.-Y., Jalilolghadr, S., Updegraff, J. A., Broström, A., \& Pakpour, A. H. (2018). Sleep hygiene behaviors in Iranian adolescents: an application of the Theory of Planned Behavior. Journal of Sleep Research, 27(1), 23-31. doi: 10.1111/jsr.12566

Vincent, N., \& Lionberg, C. (2001). Treatment preference and patient satisfaction in chronic insomnia. Sleep, 24, 411-417. doi: 10.1093/sleep/24.4.411

Yazdi, Z., Sadeghniiat-Haghighi, K., Zohal, M. A., \& Elmizadeh, K. (2012). Validity and reliability of the Iranian version of the insomnia severity index. Malaysian Journal of 
Medical Sciences, 19(4), 31-36. 
Table 1 Socio-demographic characteristics of prisoners in Rasht Lakan correctional institution $(\mathrm{N}=208)$

\begin{tabular}{lll}
\hline & Mean or $\mathrm{n}$ & SD or \% \\
\hline Age (year) & 39.08 & 7.85 \\
Educational year & 9.58 & 3.01 \\
Marital status & & \\
Single & 53 & $25.5 \%$ \\
Married & 121 & $58.2 \%$ \\
Divorced & 34 & $16.3 \%$
\end{tabular}

Having children

$\begin{array}{lll}\text { Yes } & 111 & 53.4 \% \\ \text { No } & 97 & 46.6 \%\end{array}$

Occupational status

$\begin{array}{lll}\text { Jobless } & 6 & 2.9 \% \\ \text { Daily labor } & 79 & 38.0 \% \\ \text { Self employed } & 115 & 55.3 \% \\ \text { Employed } & 8 & 3.8 \%\end{array}$

History of drug abuse

$\begin{array}{lll}\text { Yes } & 162 & 77.9 \% \\ \text { No } & 46 & 22.1 \%\end{array}$

History of alcohol abuse

$\begin{array}{lcc}\text { Yes } & 122 & 58.7 \% \\ \text { No } & 86 & 41.3 \%\end{array}$


Table 2. Pearson correlation matrix of the variables of interest

\begin{tabular}{|c|c|c|c|c|c|}
\hline & Sleep quality ${ }^{\mathrm{a}}$ & Insomnia $^{\mathrm{b}}$ & Distress $^{\mathrm{c}}$ & Stress ${ }^{\mathrm{d}}$ & Mindfulness $^{\mathrm{e}}$ \\
\hline Sleep quality & 1 & 0.675 & 0.513 & 0.389 & -0.555 \\
\hline Insomnia & & 1 & 0.589 & 0.434 & -0.557 \\
\hline Distress & & & 1 & 0.594 & -0.420 \\
\hline Stress & & & & 1 & -0.380 \\
\hline Mindfulness & & & & & 1 \\
\hline \multicolumn{6}{|c|}{${ }^{\mathrm{a}}$ Assessed using Pittsburgh Sleep Quality Index (PSQI) } \\
\hline \multicolumn{6}{|c|}{${ }^{\mathrm{b}}$ Assessed using Insomnia Severity Index (ISI) } \\
\hline \multicolumn{6}{|c|}{${ }^{\mathrm{c}}$ Assessed using Hospital Anxiety and Depression Scale (HADS) } \\
\hline \multicolumn{6}{|c|}{${ }^{\mathrm{d}}$ Assessed using Perceived Stress Scale (PSS) } \\
\hline \multicolumn{6}{|c|}{${ }^{\mathrm{e}}$ Mindful Attention Awareness Scale (MAAS) } \\
\hline Note: All $p$-va & lues $<0.01$ & & & & \\
\hline
\end{tabular}


Table 3. Mindfulness on sleep quality and insomnia with mediators of perceived stress and psychological distress

\begin{tabular}{|c|c|c|c|c|}
\hline Model & Coefficient & SE & t-value & p-value \\
\hline \multicolumn{5}{|l|}{ A. } \\
\hline Total effect of mindfulness on sleep quality (without accounting the potential mediators) & -0.144 & 0.021 & -6.73 & $<0.001$ \\
\hline Direct effect of mindfulness on sleep quality in mediated model & -0.064 & 0.020 & -3.13 & 0.002 \\
\hline Indirect effect of mindfulness on sleep quality & Effect & Boot SE & Boot LLCI & Boot ULCI \\
\hline Total indirect effect & -0.080 & 0.016 & -0.114 & -0.051 \\
\hline Indirect effect via PSS & -0.042 & 0.013 & -0.700 & -0.020 \\
\hline Indirect effect via HADS & -0.037 & 0.010 & -0.060 & -0.019 \\
\hline B. & Coefficient & SE & t-value & p-value \\
\hline Total effect of mindfulness on insomnia (without accounting the potential mediators) & -0.303 & 0.032 & -9.47 & $<0.001$ \\
\hline Direct effect of mindfulness on insomnia in mediated model & -0.174 & 0.030 & -5.84 & $<0.001$ \\
\hline Indirect effect of mindfulness on insomnia & Effect & Boot SE & Boot LLCI & Boot ULCI \\
\hline Total indirect effect & -0.128 & 0.023 & -0.174 & -0.085 \\
\hline Indirect effect via PSS & -0.038 & 0.017 & -0.074 & -0.010 \\
\hline Indirect effect via HADS & -0.090 & 0.017 & -0.124 & -0.057 \\
\hline
\end{tabular}


Boot: Bootstrapping; LLCI: lower limit confidence interval; ULCI: upper limit confidence interval; SE: standard error

Note: Age, marital status, having children, time spending in prison and years of education were adjusted for all the models.

CI 95\%, 95\% confidence interval using 5000 bootstrap samples; Boot SE, bootstrapping standard error; HADS, Hospital Anxiety and

Depression Scale; PSS, Perceived Stress Scale; MAAS, Mindful Attention Awareness Scale; Std. Coeff., standardized coefficient; Unstd. Coeff., unstandardized coefficient. 